\title{
WHITEHEAD PRODUCTS AS IMAGES OF PONTRJAGIN PRODUCTS
}

\author{
BY \\ MARTIN ARKOWITZ
}

\begin{abstract}
A method is given for computing higher order Whitehead products in the homotopy groups of a space $X$. If $X$ can be embedded in an $H$-space $E$ such that the pair $(E, X)$ has sufficiently high connectivity, then we prove that a higher order Whitehead product element in the homotopy of $X$ is the homomorphic image of a Pontrjagin product in the homology of $E$. The two main applications determine a higher order Whitehead product element in (1) $\pi_{*}\left(B U_{t}\right)$, the homotopy groups of the classifying space of the unitary group $U_{t}$, (2) the homotopy groups of a space with two nonvanishing homotopy groups.
\end{abstract}

1. Introduction. Suppose that one is interested in determining the Whitehead product $[\alpha, \beta] \in \pi_{p+q-1}(X)$ of $\alpha \in \pi_{p}(X)$ and $\beta \in \pi_{q}(X)$. If the space $X$ can be embedded in an $H$-space $E$ such that the pair $(E, X)$ is $(p+q-1)$-connected, then we prove that the Whitehead product is the homomorphic image of a Pontrjagin product in $H_{*}(E)$. More precisely, there is a homomorphism

$$
\theta: H_{p+q}(E) \rightarrow \pi_{p+q-1}(X)
$$

such that $[\alpha, \beta]=\theta(h \alpha * h \beta)$, where $h: \pi_{*}(E) \rightarrow H_{*}(E)$ is the Hurewicz homomorphism, “*” denotes Pontrjagin product in $H_{*}(E)$ and $\alpha \in \pi_{p}(X) \equiv \pi_{p}(E)$, $\beta \in \pi_{q}(X) \equiv \pi_{q}(E)$. Thus, under the hypotheses, there is a homological method for computing the Whitehead product $[\alpha, \beta]$ which depends on a knowledge of (1) the effect of $h$ on $\alpha$ and $\beta$, (2) the Pontrjagin product of $h \alpha$ and $h \beta$, (3) the homomorphism $\theta$.

The method described in the preceding paragraph is actually generalized in two ways. First, we consider $k$ th order Whitehead products instead of ordinary Whitehead products $(k=2)$; the homological approach works equally well for these higher order homotopy operations. Secondly, we require that there exists a pair $(E, A)$ with $A$ operating on $E$ instead of an $H$-space $E$. Our main theorem then yields for ordinary Whitehead products both the result of the first paragraph and a theorem of Meyer on Whitehead products and Postnikov systems. We next use the theorem to calculate some higher order Whitehead products. First we determine certain $k$ th order Whitehead product elements in $B U_{t}$, the classifying space of the

Received by the editors March 7, 1970.

AMS 1970 subject classifications. Primary 55E15; Secondary 55D45, 55F45, 55G45.

Key words and phrases. Whitehead product, higher order Whitehead product, Pontrjagin product, Postnikov systems, Samelson product, classifying space of the unitary group, Eilenberg-Mac Lane complex. 
unitary group $U_{t}$, by embedding $B U_{t}$ in the $H$-space $B U_{\infty}$ and utilizing known results on $H_{*}\left(B U_{\infty}\right)$. For $k=2$ this gives a new proof of Bott's theorem on the Samelson product $\pi_{2 r+1}\left(U_{t}\right) \otimes \pi_{2 s+1}\left(U_{t}\right) \rightarrow \pi_{2 t}\left(U_{t}\right), t=r+s+1$. Secondly, we compute $k$ th order Whitehead products in a space $X$ with two nonvanishing homotopy groups, $\pi_{n}(X)=\pi$ and $\pi_{k n-1}(X)=G$. This is achieved by embedding $X$ in the Eilenberg-Mac Lane $H$-space $K(\pi, n)$ and using known information on $H_{*}(\pi, n)$. As a corollary of this we retrieve some of Porter's results on $k$ th order Whitehead products and, for $k=2$, some results due to Meyer and Stein. As a final application we embed in $(n-1)$-connected space $X$ in $\Omega \Sigma X$. We thus obtain a new proof of the classical assertion that the Hopf invariant

$$
H: \pi_{2 n+1}(\Sigma X) \rightarrow \pi_{2 n}(\Omega \Sigma X, X)
$$

is an epimorphism if and only if the Whitehead product $\pi_{n}(X) \otimes \pi_{n}(X) \rightarrow \pi_{2 n-1}(X)$ is trivial.

I would like to express my thanks to the following person and institutions: (1) To Sufian Husseini for several informative discussions; (2) To Dartmouth College for the award of a Faculty Fellowship; (3) To the Mathematical Institute of Oxford for providing pleasant working conditions.

Many of the results of this paper were announced without proof in [1].

2. Preliminaries. We shall assume that all spaces under consideration are 1-connected pointed spaces having the homotopy type of CW-complexes. Maps and homotopies are to preserve base points. The following notation shall be used throughout this paper: $h$ for the Hurewicz homomorphism, " $\simeq$ " for the relation of homotopy between maps, $f_{\#}$ for the homomorphism of homotopy groups and $f_{*}$ for the homomorphism of homology groups induced by a map $f$, and $\partial$ for the boundary homomorphism in the exact homotopy sequence of a pair.

We now recall the definition of a $k$ th order Whitehead product (see [10]). Let $\alpha_{r} \in \pi_{n_{r}}(X), r=1, \ldots, k$, be $k$ elements in the homotopy groups of $X$. We denote the cartesian product $S^{n_{1}} \times \cdots \times S^{n_{k}}$ by $P$ and consider the following two subspaces of $P$ : (1) the lean wedge $V=S^{n_{1}} \vee \cdots \vee S^{n_{k}}$ consisting of $k$-tuples with at least $k-1$ coordinates at the base point, (2) the fat wedge $T=T\left(S^{n_{1}}, \ldots, S^{n_{k}}\right)$ consisting of $k$-tuples with at least one coordinate at the base point. Representatives of the elements $\alpha_{r}$ determine a map $g: V \rightarrow X$. We let $N=n_{1}+n_{2}+\cdots+n_{k}$ and choose a generator $\lambda$ in $H_{N}(P, T) \approx Z$ (see the proof of Theorem 3.3). If $\hat{g}: T \rightarrow X$ is an extension of $g$, then $\hat{g}_{\#} \partial h^{-1}(\lambda)$ is in $\pi_{N-1}(X)$ :

$$
H_{N}(P, T) \stackrel{h}{\stackrel{\sim}{\approx}} \pi_{N}(P, T) \stackrel{\partial}{\longrightarrow} \pi_{N-1}(T) \stackrel{\hat{g}_{\#}}{\longrightarrow} \pi_{N-1}(X) .
$$

Definition 2.1. The $k$ th order Whitehead product $\left[\alpha_{1}, \ldots, \alpha_{k}\right]$ of the elements $\alpha_{r} \in \pi_{n_{r}}(X)$ is the (possibly empty) subset $\left\{\hat{g}_{\#} \partial h^{-1}(\lambda) \mid\right.$ for every extension $\hat{g}$ of $\left.g\right\}$ of $\pi_{N-1}(X)$. 
When $k=2$ the subset $\left[\alpha_{1}, \alpha_{2}\right]$ consists of a single element, the ordinary Whitehead product of $\alpha_{1}$ and $\alpha_{2}$.

Next we consider the operation of a subspace $A$ on a space $E$.

Definition 2.2. $A$ operates on $E$ if there exists a map $\mu: E \times A \rightarrow E$ such that $\mu \mid E \simeq$ id and $\mu \mid A \simeq i$, the inclusion map $A \hookrightarrow E$.

When $A$ operates on $E$ one inductively defines maps $\mu_{n+1}: E \times A^{n} \rightarrow E$ as follows: Let $\mu_{2}=\mu$; assume $\mu_{n}$ already defined and let $\mu_{n+1}$ be the composition

$$
E \times A^{n}=\left(E \times A^{n-1}\right) \times A \stackrel{\mu_{n} \times \mathrm{id}}{\longrightarrow} E \times A \stackrel{\mu}{\longrightarrow} E .
$$

Clearly $\mu_{n+1} \mid E \simeq$ id and $\mu_{n+1} \mid$ each $A \simeq i$.

Definition 2.3. If $A$ operates on $E$, then $\mu_{k}: E \times A^{k-1} \rightarrow E$ induces a $k$-fold generalized Pontrjagin product

$$
H_{n_{1}}(E) \otimes H_{n_{2}}(A) \otimes \cdots \otimes H_{n_{k}}(A) \hookrightarrow H_{n_{1}+\cdots+n_{k}}\left(E \times A^{k-1}\right) \stackrel{\mu_{k *}}{\longrightarrow} H_{n_{1}+\cdots+n_{k}}(E) .
$$

If $a_{1} \in H_{n_{1}}(E)$ and $a_{r} \in H_{n_{r}}(A), r=2, \ldots, k$, then $\mu_{k *}\left(a_{1} \otimes a_{2} \otimes \cdots \otimes a_{k}\right)$ is denoted by $a_{1} * a_{2} * \cdots * a_{k}$.

If $E$ is an $H$-space then $E$ operates on $E$ with $\mu: E \times E \rightarrow E$ the multiplication. In this case the $k$-fold generalized Pontrjagin product is just the $k$-fold ordinary Pontrjagin product.

3. The main theorem. We state the hypotheses of our main theorem which enables us to compute a $k$ th order Whitehead product element of elements in $\pi_{n_{r}}(X), r=1, \ldots, k$.

Hypothesis of TheOrem 3.3. (1) There exists a pair of spaces $(E, A)$ such that $A$ operates on $E$ and the inclusion map $i: A \rightarrow E$ induces an isomorphism $i_{\#}: \pi_{s}(A) \rightarrow \pi_{s}(E)$ for $s=n_{2}, \ldots, n_{k}$.

(2) There exists a map $f: X \rightarrow E$ such that $f_{\#}: \pi_{s}(X) \rightarrow \pi_{s}(E)$ is an isomorphism for $s<N-1$ and an epimorphism for $s=N-1$, where $N=n_{1}+n_{2}+\cdots+n_{k}$.

Without loss of generality we will assume that the $\operatorname{map} f: X \rightarrow E$ is an inclusion, for we can always replace $E$ by the mapping cylinder of $f$. Then by Hypothesis (2), the pair $(E, X)$ is $(N-1)$-connected and so the Hurewicz homomorphism $h: \pi_{N}(E, X) \rightarrow H_{N}(E, X)$ is an isomorphism.

Definition 3.1. Under Hypothesis (2) of Theorem 3.3 a homomorphism $\theta: H_{N}(E) \rightarrow \pi_{N-1}(X)$ is defined by $\theta=\partial h^{-1} j$, where $j$ is induced by inclusion:

$$
H_{N}(E) \stackrel{j}{\longrightarrow} H_{N}(E, X) \stackrel{h^{-1}}{\approx} \pi_{N}(E, X) \stackrel{\partial}{\longrightarrow} \pi_{N-1}(X) .
$$

REMARK 3.2. We observe (but do not prove) that $\theta$ has an alternate description. Replace $f: X \rightarrow E$ by a fibre map and let $F$ denote the fibre. Thus $F \rightarrow X \stackrel{f}{\rightarrow} E$ is essentially a fibre sequence and $F$ is clearly $(N-2)$-connected. Then $\theta$ can be shown to be the composition

$$
H_{N}(E) \stackrel{\tau}{\longrightarrow} H_{N-1}(F) \stackrel{h^{-1}}{\underset{\approx}{\longrightarrow}} \pi_{N-1}(F) \longrightarrow \pi_{N-1}(X)
$$


where $\tau$ is the transgression homomorphism of the fibration and the homomorphism on the right is induced by inclusion.

Next we state and prove Theorem 3.3. By Hypothesis (2) $f_{\#}: \pi_{n_{r}}(X) \rightarrow \pi_{n_{r}}(E)$ is an isomorphism for $r=1, \ldots, k$. We will identify these groups and write ambiguously $\alpha_{r} \in \pi_{n_{r}}(X) \equiv \pi_{n_{r}}(E)$.

THEOREM 3.3. Under the Hypotheses (1) and (2) above, the kth order Whitehead product set $\left[\alpha_{1}, \ldots, \alpha_{k}\right] \subset \pi_{N-1}(X)$ of elements $\alpha_{r} \in \pi_{n_{r}}(X), r=1, \ldots, k$, is nonempty and one of its elements is

$$
\theta\left(h\left(\alpha_{1}\right) * h\left(i_{\#}^{-1} \alpha_{2}\right) * \cdots * h\left(i_{\#}^{-1} \alpha_{k}\right)\right)
$$

where $*$ denotes the generalized Pontrjagin product.

Proof. Let the $\alpha_{r}$ be represented by maps $a_{r}: S^{n_{r}} \rightarrow X$. By Hypothesis (1) there exist maps $a_{r}^{\prime}: S^{n_{r}} \rightarrow A$ such that $i a_{r}^{\prime} \simeq f a_{r}$ for $r=2, \ldots, k$. The maps $a_{1}, a_{2}, \ldots, a_{k}$ determine a map $g: V=S^{n_{1}} \vee \cdots \vee S^{n_{k}} \rightarrow X$ and a map $\tilde{g}: P=S^{n_{1}} \times \cdots \times S^{n_{k}} \rightarrow E$ defined by $\tilde{g}=\mu_{k}\left(f a_{1} \times a_{2}^{\prime} \times \cdots \times a_{k}^{\prime}\right)$ :

$$
S^{n_{1}} \times S^{n_{2}} \times \cdots \times S^{n_{k}} \stackrel{f a_{1} \times a_{2}^{\prime} \times \cdots \times a_{k}^{\prime}}{\longrightarrow} E \times A^{k-1} \longrightarrow \mu_{k} \longrightarrow E .
$$

Then there is homotopy-commutativity in the diagram

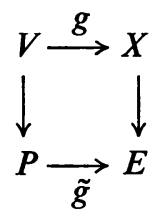

and by the homotopy extension property of $(P, V)$ we replace $\tilde{g}$ by a homotopic map (also called $\tilde{g}$ ) such that the above square commutes. Restrict $\tilde{g}$ to the fat wedge $T$ to obtain a map of pairs $\tilde{g}: T, V \rightarrow E, X$. The obstructions to deforming this map into $X$ lie in the group $H^{i}\left(T, V ; \pi_{i}(E, X)\right)$ (see [7, p. 197]). But $(E, X)$ has trivial homotopy in dimensions $<N$ and for dimension reasons $(T, V)$ has trivial cohomology in dimensions $\geqq N$. Therefore there exists a map $\hat{g}: T \rightarrow X$ such that in the following diagram

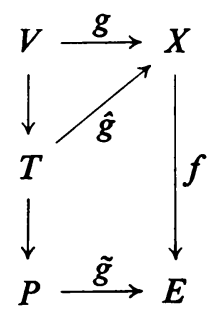

the triangle commutes and the trapezoid homotopy commutes. Here again we use the homotopy extension property and replace $\tilde{g}$ by a homotopic map such that the 
trapezoid commutes. Then $\hat{g}: T \rightarrow X$ is an extension of $g: V \rightarrow X$ and hence a Whitehead product element

$$
\hat{g}_{\#} \partial h^{-1}(\lambda) \in\left[\alpha_{1}, \ldots, \alpha_{k}\right] \subset \pi_{N-1}(X)
$$

exists.

Now we prove that this element is $\theta\left(h \alpha_{1} * h i_{\#}^{-1} \alpha_{2} * \cdots * h i_{\#}^{-1} \alpha_{k}\right)$. Consider the commutative diagram

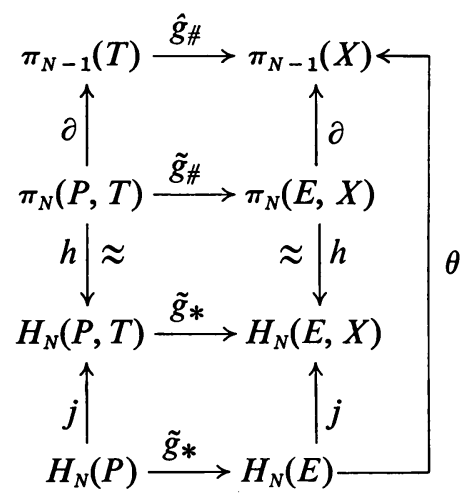

For the generator $\lambda \in H_{N}(P, T)$ we choose $j\left(s_{1} \otimes \cdots \otimes s_{k}\right)$ where $s_{r} \in H_{n_{r}}\left(S^{n_{r}}\right)$ is the standard generator and $s_{1} \otimes \cdots \otimes s_{k} \in H_{n_{1}}\left(S^{n_{1}}\right) \otimes \cdots \otimes H_{n_{r}}\left(S^{n_{r}}\right) \equiv H_{N}(P)$. Then

$$
\hat{g}_{\#} \partial h^{-1}(\lambda)=\partial h^{-1} \tilde{g}_{*}(\lambda)=\partial h^{-1} j \tilde{g}_{*}\left(s_{1} \otimes \cdots \otimes s_{k}\right)=\theta \tilde{g}_{*}\left(s_{1} \otimes \cdots \otimes s_{k}\right) .
$$

But $\tilde{g} \simeq \mu_{k}\left(f a_{1} \times a_{2}^{\prime} \times \cdots \times a_{k}^{\prime}\right): P \rightarrow E$ and so

$$
\begin{aligned}
\theta \tilde{g}_{*}\left(s_{1} \otimes \cdots \otimes s_{k}\right) & =\theta \mu_{k *}\left(\left(f a_{1}\right)_{*}\left(s_{1}\right) \otimes a_{2 *}^{\prime}\left(s_{2}\right) \otimes \cdots \otimes a_{k *}^{\prime}\left(s_{k}\right)\right) \\
& =\theta\left(h\left(\alpha_{1}\right) * h\left(i_{\#}^{-1} \alpha_{2}\right) * \cdots * h\left(i_{\#}^{-1} \alpha_{k}\right)\right) .
\end{aligned}
$$

For the first corollary of the theorem assume that $Y$ is a $(p-1)$-connected space and $2 \leqq p \leqq q$. Let $Y_{n}$ denote the $n$th Postnikov section of $Y$ and let $Y_{q, p+q-2}$ denote the fibre of $Y_{p+q-2} \rightarrow Y_{q-1}$. Since this fibration is principal there is an action of $Y_{q, p+q-2}$ on $Y_{p+q-2}$. We let $X=Y_{p+q-1}, A=Y_{q, p+q-2}, E=Y_{p+q-2}$ and $f: X \rightarrow E$ be the fibre map. The hypotheses of Theorem 3.3 are then satisfied for the ordinary Whitehead product of $\alpha \in \pi_{p}(Y) \equiv \pi_{p}(X)$ and $\beta \in \pi_{q}(Y) \equiv \pi_{q}(X)$. We obtain as a corollary Meyer's theorem [8].

Corollary 3.4. $[\alpha, \beta]=\theta\left(h(\alpha) * h\left(i_{\#}^{-1} \beta\right)\right)$.

By Remark 3.2, $\theta: H_{p+q}\left(Y_{p+q-2}\right) \rightarrow \pi_{p+q-1}\left(Y_{p+q-1}\right)=\pi_{p+q-1}(Y)$ can be identified with the transgression homomorphism

$$
H_{p+q}\left(Y_{p+q-2}\right) \rightarrow H_{p+q-1}\left(F_{p+q-1}\right) \equiv \pi_{p+q-1}\left(F_{p+q-1}\right)=\pi_{p+q-1}(Y)
$$

of the fibration $F_{p+q-1} \rightarrow Y_{p+q-1} \rightarrow Y_{p+q-2}$.

For applications in the next section we will need the following corollary. 
Corollary 3.5. Suppose there exists a map of $X$ into an $H$-space $E$ such that $\pi_{s}(X) \rightarrow \pi_{s}(E)$ is an isomorphism for $s<N-1$ and an epimorphism for $s=N-1$, where $N=n_{1}+\cdots+n_{k}$. Then, for $\alpha_{r} \in \pi_{n_{r}}(X) \equiv \pi_{n_{r}}(E)$.

$$
\theta\left(h \alpha_{1} * \ldots * h \alpha_{k}\right) \in\left[\alpha_{1}, \ldots, \alpha_{k}\right] \subset \pi_{N-1}(X)
$$

where $*$ denotes the ordinary Pontrjagin product.

4. Applications. In this section we use Corollary 3.5 to compute certain higher order Whitehead product elements. Our first result concerns Whitehead products in $\pi_{*}\left(B U_{t}\right)$, where $B U_{t}$ is the classifying space of $U_{t}$, the unitary group of $t \times t$ matrices. Recall that for $i<2 t+1, \pi_{i}\left(B U_{t}\right)$ is either 0 or $Z$ depending on whether $i$ is odd or even, and that $\pi_{2 t+1}\left(B U_{t}\right) \approx Z_{t !}$.

THEOREM 4.1. If $\alpha_{r} \in \pi_{2 m_{r}+2}\left(B U_{t}\right) \approx Z$ and $\gamma \in \pi_{2 t+1}\left(B U_{t}\right) \approx Z_{t !}$ are suitable generators, $r=1,2, \ldots, k$ and $t=m_{1}+\cdots+m_{k}+k-1$, then

$$
m_{1} ! \cdots m_{k} ! \gamma \in\left[\alpha_{1}, \ldots, \alpha_{k}\right] \subset \pi_{2 t+1}\left(B U_{t}\right) .
$$

Proof. We write $B_{n}$ for $B U_{n}, 1 \leqq n \leqq \infty$, and let $f: B_{t} \rightarrow B_{\infty}$ be the inclusion map. It is well known that $B_{\infty}$ is an $H$-space and that $f$ is an isomorphism of homotopy groups in dimensions $<2 t+1=N-1$ and an epimorphism in dimension $2 t+1$ $=N-1$. By Corollary 3.5, the Whitehead product set $\left[\alpha_{1}, \ldots, \alpha_{k}\right] \subset \pi_{N-1}\left(B_{t}\right)$ is nonempty and one of its elements is $\theta\left(h \alpha_{1} * \cdots * h \alpha_{k}\right)$, where $h \alpha_{r} \in H_{2 m_{r}+2}\left(B_{\infty}\right)$ is the Hurewicz homomorphism image of $\alpha_{r} \in \pi_{2 m_{r}+2}\left(B_{\infty}\right)$ and $\theta$ is the composition

$$
H_{N}\left(B_{\infty}\right) \stackrel{j}{\longrightarrow} H_{N}\left(B_{\infty}, B_{t}\right) \stackrel{h^{-1}}{\underset{\approx}{\longrightarrow}} \pi_{N}\left(B_{\infty}, B_{t}\right) \stackrel{\partial}{\longrightarrow} \pi_{N-1}\left(B_{t}\right) .
$$

It remains to show that $\theta\left(h \alpha_{1} * \cdots * h \alpha_{k}\right)=m_{1} ! \cdots m_{k} ! \gamma$.

We recall some known facts on the homology and homotopy of $B_{n}$. The general reference is [5], especially 11-12-11-13 and 17-01-17-03. The multiplication in $H_{*}\left(B_{\infty}\right)$ (i.e., the Pontrjagin product) is denoted by $*$ or by juxtaposition or by a dot.

(1) The homomorphism $\partial: \pi_{N}\left(B_{\infty}, B_{t}\right) \rightarrow \pi_{N-1}\left(B_{t}\right)$ is the projection $Z \rightarrow Z_{t}$, for $\pi_{N}\left(B_{\infty}\right) \rightarrow \pi_{N}\left(B_{\infty}, B_{t}\right)$ is the homomorphism $Z \rightarrow Z$ consisting of multiplication by $t$ !.

(2) $h\left(\alpha_{r}\right)=m_{r} ! p_{2 m_{r}+2}$, where $p_{2 i} \in H_{2 i}\left(B_{\infty}\right)$ is the generator in dimension $2 i$ of the primitive elements of the Hopf algebra $H_{*}\left(B_{\infty}\right)$.

(3) $H_{*}\left(B_{\infty}\right)$ is a polynomial algebra on generators $x_{2}, x_{4}, x_{6}, \ldots$, where degree $x_{2 i}=2 i$. Furthermore $p_{2 m_{1}+2} \cdot p_{2 m_{2}+2} \cdots \cdots p_{2 m_{k}+2}=x_{2}^{t+1}+$ linear combination of other monomials in the $x$ 's. This last assertion is an easy consequence of the following result $[5,17-03]$ :

$$
p_{2}=x_{2}, \ldots, p_{2 i}=(-1)^{i+1} i x_{2 i}+\sum_{j=1}^{i-1}(-1)^{j+1} x_{2 j} p_{2 i-2 j}, \ldots
$$

(4) The homomorphism $j: H_{N}\left(B_{\infty}\right) \rightarrow H_{N}\left(B_{\infty}, B_{t}\right)$ maps all monomials $x_{2 k_{1}} \cdots \cdots x_{2 k_{s}}\left(2 k_{1}+\cdots+2 k_{s}=N\right)$ except $x_{2}^{t+1}$ to zero and maps $x_{2}^{t+1}$ onto a generator of $H_{N}\left(B_{\infty}, B_{t}\right)=Z$. 
We put these facts together and obtain

$$
\begin{aligned}
\theta\left(h \alpha_{1} * \cdots * h \alpha_{k}\right) & =m_{1} ! \cdots m_{k} ! \theta\left(p_{2 m_{1}+2} \cdots \cdots p_{2 m_{k}+2}\right) \\
& =m_{1} ! \cdots m_{k} ! \theta\left(x_{2}^{t+1}+\text { other monomials }\right) \\
& =m_{1} ! \cdots m_{k} ! \partial h^{-1} j\left(x_{2}^{t+1}\right) .
\end{aligned}
$$

But $j\left(x_{2}^{t+1}\right)$ is a generator of $H_{N}\left(B_{\infty}, B_{t}\right) \approx Z$ by (4) and so $\partial h^{-1} j\left(x_{2}^{t+1}\right)$ is a generator $\gamma$ of $\pi_{N-1}\left(B_{t}\right) \approx Z_{t !}$ by (1). Thus $m_{1} ! \cdots m_{k} ! \gamma \in\left[\alpha_{1}, \ldots, \alpha_{k}\right]$.

REMARK 4.2. For $k=2$, Theorem 4.1 provides a new proof of Bott's theorem [2] on the Whitehead product $\pi_{2 r+2}\left(B U_{t}\right) \otimes \pi_{2 s+2}\left(B U_{t}\right) \rightarrow \pi_{2 t+1}\left(B U_{t}\right)$ (or what is the same thing, the Samelson product $\left.\pi_{2 r+1}\left(U_{t}\right) \otimes \pi_{2 s+1}\left(U_{t}\right) \rightarrow \pi_{2 t}\left(U_{t}\right)\right)$, where $t=r+s+1$. In addition, we can similarly prove a result which is completely analogous to Theorem 4.1 for the symplectic group $S p_{t}$. In this way we compute a higher order Whitehead product element in $\pi_{*}\left(B S p_{t}\right)$ and obtain another proof of Bott's theorem on a Samelson product in $\pi_{*}\left(S p_{t}\right)$.

Our next application of Corollary 3.5 concerns $k$-fold Whitehead products in a space $X$ such that $\pi_{i}(X)=0$ for $i<n$ and $n<i<k n-1$, where $n>1$. Let $\pi_{n}(X)=\pi$ and $\pi_{k n-1}(X)=G$ and let $l \in H^{k n}(\pi, n ; G)$ denote the first Postnikov invariant of $X$ and $\gamma_{k}: H_{n}(\pi, n)=\pi \rightarrow H_{k n}(\pi, n)$ be the $k$ th divided power in the ring $H_{*}(\pi, n)$ [4].

THEOREM 4.3. Let $X$ satisfy the conditions of the preceding paragraph. If $\alpha \in \pi_{n}(X)=\pi$ and $s_{1}, \ldots, s_{k}$ are any $k$ integers, then the $k$ th order Whitehead product $\left[s_{1} \alpha, \ldots, s_{k} \alpha\right]$ is a unique element of $\pi_{k n-1}(X)=G$.

(a) If $n$ is odd, $\left[s_{1} \alpha, \ldots, s_{k} \alpha\right]=0$.

(b) If $n$ is even, $\left[s_{1} \alpha, \ldots, s_{k} \alpha\right]=s_{1} \cdots s_{k} k ! l_{*} \gamma_{k}(\alpha)$, where $l_{*}: H_{k n}(\pi, n) \rightarrow H_{k n}(G, k n)$ $=G$ is induced by $l$.

Proof. We embed $X$ in an Eilenberg-Mac Lane complex $K(\pi, n)$ in the usual way. Clearly $K(\pi, n)$ is an $H$-space and the map $X \rightarrow K(\pi, n)$ satisfies the hypotheses of Corollary 3.5. Then the $k$ th order Whitehead product $\left[s_{1} \alpha, \ldots, s_{k} \alpha\right]$ exists and one of its elements is $s_{1} \cdots s_{k} \theta(h \alpha * \cdots * h \alpha)$. We show first that this Whitehead product element is unique. If there are two extensions of a map $V=S^{n} \vee \cdots \vee S^{n} \rightarrow X$ to the fat wedge $T=T\left(S^{n}, \ldots, S^{n}\right)$ then the obstructions to their being homotopic lie in the groups $H^{i}\left(T, V ; \pi_{i}(X)\right)$ which are always zero. Thus there is up to homotopy exactly one extension of a map $V \rightarrow X$ to $T$. Therefore $\left[s_{1} \alpha, \ldots, s_{k} \alpha\right]$ consists of the element $s_{1} \cdots s_{k} \theta(h \alpha * \cdots * h \alpha)$. Here $\alpha \in \pi=\pi_{n}(X)$ $\equiv \pi_{n}(K(\pi, n)) \stackrel{h}{\stackrel{h}{\sim}} H_{n}(\pi, n)=\pi$ and we shall write $\alpha$ for the element in each of these groups (identifying $\alpha$ with $h \alpha)$. Thus we must determine $\theta(\alpha * \cdots * \alpha)=\theta\left(\alpha^{k}\right)$ for $\alpha \in H_{n}(\pi, n)$. We recall the following known facts about $H_{*}(\pi, n)$ (the general reference is [4]). Let $U(\pi, n)$ be the universal graded strictly anticommutative algebra with divided powers generated by the graded abelian group which is trivial in dimensions $\neq n$ and is $\pi$ in dimension $n$. Then the subalgebra with divided powers generated by $H_{n}(\pi, n)=\pi$ is isomorphic to $U(\pi, n)$. If $n$ is odd, $U(\pi, n)$ is the exterior algebra of $\pi$, suitably graded. Hence $\alpha^{k}=\alpha * \cdots * \alpha=0$ for 
$n$ odd, and so (a) is proved. To prove (b), we note that $\alpha^{k}=\left(\gamma_{1}(\alpha)\right)^{k}=k ! \gamma_{k}(\alpha)$ and so $\theta\left(\alpha^{k}\right)=k ! \theta\left(\gamma_{k}(\alpha)\right)$. We must show that under appropriate identification the homomorphism $\theta: H_{k n}(\pi, n) \rightarrow \pi_{k n-1}(X)=G$ is just the homomorphism $l_{*}: H_{k n}(\pi, n) \rightarrow H_{k n}(G, k n)=G$. This follows easily from Remark 3.2, but we will establish it directly. If $X_{i}$ denotes the $i$ th Postnikov section of $X$ then $X_{n}=K(\pi, n)$. We denote $K(G, k n)$ by $K$ and regard maps between spaces as inclusions whenever convenient. First we identify $j: H_{k n}\left(X_{n}\right) \rightarrow H_{k n}\left(X_{n}, X\right)$ with

$$
j: H_{k n}\left(X_{n}\right) \rightarrow H_{k n}\left(X_{n}, X_{k n-1}\right),
$$

for the inclusion map $\left(X_{n}, X\right) \rightarrow\left(X_{n}, X_{k n-1}\right)$ induces an isomorphism $H_{k n}\left(X_{n}, X\right)$ $\underset{\approx}{\rightarrow} H_{k n}\left(X_{n}, X_{k n-1}\right)$ (e.g., by the five lemma). But $X_{k n-1} \rightarrow X_{n} \stackrel{l}{\rightarrow} K$ is essentially a fibre sequence and so $H_{k n}\left(X_{n}, X_{k n-1}\right) \rightarrow H_{k n}(K)$ is an isomorphism (either by the Serre theorem or by arguing on the homotopy groups and then passing to homology). Thus we have the following commutative diagram:

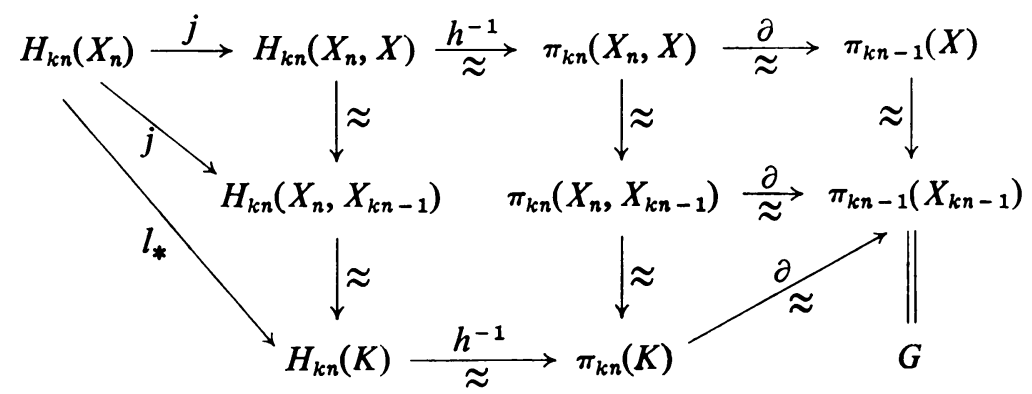

Since the top line $\partial h^{-1} j$ is just $\theta$, under the identification of $\pi_{k n-1}(X)$ with $G$ and of $H_{k n}(K)$ with $G$ via $\partial h^{-1}, \theta: H_{k n}\left(X_{n}\right) \rightarrow G$ is identified with $l_{*}: H_{k n}\left(X_{n}\right)$ $=H_{k n}(\pi, n) \rightarrow H_{k n}(K)=G$. This completes the proof.

In the case when $n$ is even, more complete information can be obtained when $\pi$ and $G$ are the group of integers.

Corollary 4.4. Let $X$ satisfy the hypothesis of Theorem 4.3 with $G=\pi=Z$. Let the first Postnikov invariant $l=m b^{k}$, a multiple of the kth cup product of the basic class $b \in H^{n}(Z, n)$. Let $\alpha \in \pi$ and $\gamma \in G$ be generators. Then if $n$ is even and $s_{1}, \ldots, s_{k}$ are any integers,

$$
\left[s_{1} \alpha, \ldots, s_{k} \alpha\right]=m k ! s_{1} \cdots \cdots s_{k} \gamma .
$$

The corollary follows immediately from the fact that $\left(b^{k}\right)_{*}\left(\gamma_{k}(\alpha)\right) \in H_{k n}(Z, k n)$ $=Z$ is a generator.

REMARKS 4.5. (a) Porter's result [10] on the $k$ th order Whitehead product in complex projective $(k-1)$-space follows from Corollary 4.4 by setting $n=2$ and $m=1$. 
(b) The last two results provide another way to obtain some of Porter's examples for certain phenomena regarding higher order Whitehead products [9]. For example, by Corollary 4.4 there is for every $k$ a space in which all $i$ th order Whitehead products are trivial, $i<k$, and in which there exists a nontrivial $k$ th order Whitehead product. Also, by Theorem 4.3, one can easily find spaces in which all $k$ th order Whitehead products vanish, but which are not $H$-spaces.

(c) For $k=2$, Theorem 4.3 yields a result on ordinary Whitehead products which is similar to but somewhat weaker than a result of Stein and Meyer [11]. Our proof for $k=2$ is not substantially different from that of [11]. We note that one direction of Stein's theorem can be generalized to higher order Whitehead products as follows: Let $X$ satisfy the hypothesis of Theorem 4.3 with $n$ even. Then there exists a function $\mathscr{K}: \pi \rightarrow G$ such that $\mathscr{K}(s \alpha)=s^{k} \mathscr{K}(\alpha)$ and

$$
\begin{gathered}
{\left[\alpha_{1}, \ldots, \alpha_{k}\right]=\mathscr{K}\left(\alpha_{1}+\cdots+\alpha_{k}\right)-\sum_{i=0}^{k} \mathscr{K}\left(\alpha_{1}+\cdots+\hat{\alpha}_{i}+\cdots+\alpha_{k}\right)} \\
+\cdots+(-1)^{k-1}\left(\mathscr{K}\left(\alpha_{1}\right)+\cdots+\mathscr{K}\left(\alpha_{k}\right)\right)
\end{gathered}
$$

for any $\alpha_{i} \in \pi$. The proof proceeds just like the proof of Theorem 4.3 with $\mathscr{K}=l_{*} \gamma_{k}$.

Despite the fact that proof of Corollary 3.5 is not difficult, the result appears to be new even for ordinary Whitehead products (see the first paragraph of $\S 1$ for an explicit statement in that case). We next use Corollary 3.5 to derive a few classical results on ordinary Whitehead products.

Proposition 4.6. If $X$ is an $H$-space, then all ordinary Whitehead products in $X$ vanish.

For we take $X=E$ and note that $\theta$ factors through $H_{*}(X, X)$.

Our final application is the theorem relating the vanishing of Whitehead products to the existence of elements of Hopf invariant one.

Let $X$ be an $(n-1)$-connected space, $n>1$. Let $e: X \rightarrow \Omega \Sigma X$ denote the canonical embedding of $X$ into the loop space of the suspension of $X$ and let $j: \Omega \Sigma X \rightarrow \Omega \Sigma X, X$ be the inclusion map. Then the generalized Hopf invariant homomorphism $H: \pi_{2 n+1}(\Sigma X) \rightarrow \pi_{2 n}(\Omega \Sigma X, X)$ has been defined (see e.g. [4, Exposé 22]) as the composition

$$
\pi_{2 n+1}(\Sigma X) \approx \pi_{2 n}(\Omega \Sigma X) \stackrel{j_{\#}}{\longrightarrow} \pi_{2 n}(\Omega \Sigma X, X) .
$$

In the case $X=S^{n}$ this is just a homomorphism $H: \pi_{2 n+1}\left(S^{n+1}\right) \rightarrow \pi_{2 n}\left(\Omega S^{n+1}, S^{n}\right)$ $=Z$. The following theorem is known.

THEOREM 4.7. The generalized Hopf invariant $H: \pi_{2 n+1}(\Sigma X) \rightarrow \pi_{2 n}(\Omega \Sigma X, X)$ of an $(n-1)$-connected space $X$ is an epimorphism if and only if $[\alpha, \beta]=0$ for every $\alpha, \beta \in \pi_{n}(X)$. 
Proof. $\Omega \Sigma X$ is an $H$-space and $e: X \rightarrow \Omega \Sigma X$ satisfies the hypothesis of Corollary 3.5 with $k=2$ and $n_{1}=n_{2}=n$. Therefore $[\alpha, \beta]=\theta(h \alpha * h \beta)=\theta\left(e_{*} \alpha * e_{*} \beta\right)$ where $\alpha, \beta \in \pi_{n}(X) \equiv \pi_{n}(\Omega \Sigma X)$ are identified with their Hurewicz images in $H_{n}(X)$. Consider the diagram

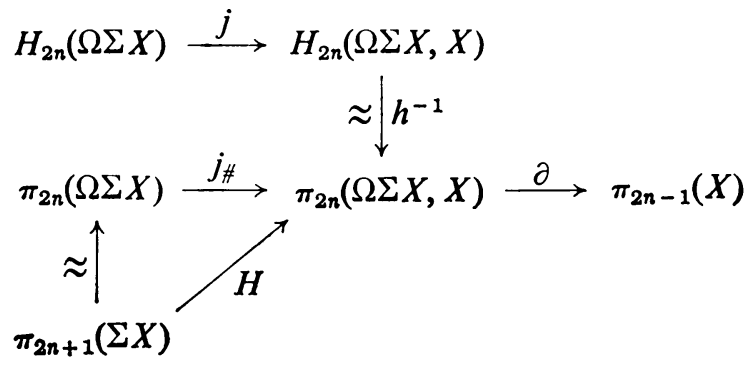

It is not difficult to show that $H_{2 n}(\Omega \Sigma X, X)$ consists of elements of the form $j\left(e_{*} \alpha * e_{*} \beta\right)$ for every $\alpha, \beta \in H_{n}(X)$. When $H_{*}(X)$ is torsion-free this follows from the Bott-Samelson theorem [3] which expresses the Pontrjagin algebra $H_{*}(\Omega \Sigma X)$ as a tensor algebra on $e_{*} H_{+}(X)$. In general a theorem of Copeland enables one to express $H_{*}(\Omega \Sigma X)$ as a torsion-tensor algebra on $e_{*} H_{+}(X)$. In any case $H_{2 n}(\Omega \Sigma X)$ $\approx H_{2 n}(X) \oplus H_{n}(X) \otimes H_{n}(X)$. Thus $\pi_{2 n}(\Omega \Sigma X, X)$ consists of all elements of the form $h^{-1} j\left(e_{*} \alpha * e_{*} \beta\right)$ and $[\alpha, \beta]=\partial h^{-1} j\left(e_{*} \alpha * e_{*} \beta\right)$. Hence $[\alpha, \beta]=0$ for every $\alpha$ and $\beta$ if and only if $\partial=0$. By exactness this is so if and only if $j_{\#}$ and consequently $H$ is an epimorphism.

COROLlaRY $4.8[12,3.49]$. Let $\iota_{n} \in \pi_{n}\left(S^{n}\right)$ be the class of the identity map. Then $\left[\iota_{n}, \iota_{n}\right]=0 \in \pi_{2 n-1}\left(S^{n}\right)$ if and only if there is an element in $\pi_{2 n+1}\left(S^{n+1}\right)$ of Hopf invariant one.

\section{REFERENCES}

1. M. Arkowitz, A homological method for computing certain Whitehead products, Bull. Amer. Math. Soc. 74 (1968), 1079-1082. MR 39 \#2159.

2. R. Bott, A note on the Samelson product in the classical groups, Comment. Math. Helv. 34 (1960), 249-256. MR 23 \#A658.

3. R. Bott and H. Samelson, On the Pontryagin product in spaces of paths, Comment. Math. Helv. 27 (1953), 320-337. MR 15, 643.

4. Séminaire Henri Cartan École Norm. Sup. 1954/55, Secrétariat mathématique, Paris, 1955. MR 19, 438.

5. Séminaire Henri Cartan 1959/60, École Norm. Sup., Secrétariat mathématique, Paris, 1961. MR $28 \# 1092$.

6. A. H. Copeland, Jr., The Pontrjagin ring for certain loop spaces, Proc. Amer. Math. Soc. 7 (1956), 528-534. MR 17, 1232.

7. S. T. Hu, Homotopy theory, Pure and Appl. Math., vol. 8, Academic Press, New York, 1959. MR 21 \#5186.

8. J.-P. Meyer, Whitehead products and Postnikov systems, Amer. J. Math. 82 (1960), 271-280. MR 26 \#6965. 
9. G. J. Porter, Spaces with vanishing Whitehead products, Quart. J. Math. Oxford Ser. (2) 16 (1965), 77-84. MR 30 \#2511.

10. - Higher order Whitehead products and Postnikov systems, Illinois J. Math. 11 (1967), 414-416. MR 36 \#7138.

11. N. Stein, Note on the realization of Whitehead products, Quart. J. Math. Oxford Ser. (2) 17 (1966), 160-164. MR 33 \#4932.

12. G. W. Whitehead, A generalization of the Hopf invariant, Ann. of Math. (2) 51 (1950), 192-237. MR 12, 847.

Dartmouth College,

HANOVER, New HAMPSHIRE 03755 